\title{
Melting of “Porous” Vortex Matter
}

\author{
S. S. Banerjee, ${ }^{1}$ A. Soibel,,${ }^{1 *}$ Y. Myasoedov, ${ }^{1}$ M. Rappaport,,${ }^{1}$ E. Zeldov, ${ }^{1}$ M. Menghini, ${ }^{2}$ Y. Fasano, ${ }^{2}$ F. de la Cruz, ${ }^{2}$ \\ C. J. van der Beek, ${ }^{3}$ M. Konczykowski, ${ }^{3}$ and T. Tamegai ${ }^{4}$ \\ ${ }^{1}$ Department of Condensed Matter Physics, Weizmann Institute of Science, Rehovot 76100, Israel \\ ${ }^{2}$ Instituto Balseiro and Centro Atómico Bariloche, CNEA, Avenue Bustillo 9500, Bariloche, RN, Argentina \\ ${ }^{3}$ Laboratoire des Solides Irradiés, CNRS UMR 7642, Ecole Polytechnique, 91128 Palaiseau, France \\ ${ }^{4}$ Department of Applied Physics, University of Tokyo, Hongo, Bunkyo-ku, Tokyo 113-8656, \\ and CREST Japan Science and Technology Corporation (JST), Japan
}

(Received 16 July 2002; published 28 February 2003)

\begin{abstract}
Bitter decoration and magneto-optical studies reveal that in heavy-ion irradiated superconductors, a "porous" vortex matter is formed when vortices outnumber columnar defects. In this state ordered vortex crystallites are embedded in the "pores" of a rigid matrix of vortices pinned on columnar defects. The crystallites melt through a first-order transition while the matrix remains solid. The melting temperature increases with density of columnar defects and eventually turns into a continuous transition. At high temperatures a sharp kink in the melting line is found, signaling an abrupt change from crystallite melting to melting of the rigid matrix.
\end{abstract}

DOI: 10.1103/PhysRevLett.90.087004

Melting of heterogeneous systems, and, in particular, of nanocrystals embedded in porous rigid matrices, is a complex process with many uncontrolled parameters. Metal and semiconductor nanocrystals with free surfaces usually show a decrease in their melting temperature $T_{m}$ with decreasing size [1], whereas nanocrystals encapsulated in a porous matrix often display an increase in $T_{m}$ [2]. This melting process is known to depend on the size, dimensionality, material properties of the nanocrystals and the matrix, as well as the interface energies between the materials $[1,2]$. In this work we investigate an analogous, but more controllable composite system, which is a "porous" vortex matter consisting of vortex nanocrystals encapsulated in a matrix of strongly pinned vortices. As shown below, this system is present in the commonly heavy-ion irradiated superconductors when the vortices outnumber the columnar defects (CDs). The rigid matrix is created by vortices localized on the network of random CDs, while the softer nanocrystals are formed within the pores of this matrix by the interstitial vortices. The size of the nanocrystals can be readily varied from several hundred down to a few vortices by changing the applied field or the density of CDs. We find that this composite vortex matter reveals a number of intriguing mechanisms: Similarly to the metallic nanocrystals in a matrix, we observe for the first time a pronounced upward shift in the vortex melting temperature $T_{m}$, while preserving the first-order nature of the transition (FOT). With increasing density of CDs, the size of the pores decreases, resulting in a larger shift in $T_{m}$. We also find a critical point at which the FOT changes into a continuous melting. Moreover, the crystallites can melt while the matrix remains rigid, giving rise to an abrupt breakdown in the upward shift of $T_{m}$ and a sharp kink in the FOT line.

The reported findings were obtained using Bitter decoration and differential magneto-optical (MO) [3] tech-
PACS numbers: 74.25.Op, 74.25.Qt, 74.72.Hs, 74.81.-g

niques. High quality $\mathrm{Bi}_{2} \mathrm{Sr}_{2} \mathrm{CaCu}_{2} \mathrm{O}_{8}$ (BSCCO) crystals $\left(T_{c} \approx 89 \mathrm{~K}\right)$ were irradiated by $1 \mathrm{GeV} \mathrm{Pb}$ ions through various patterned masks at GANIL, with doses corresponding to matching fields of $B_{\phi}=5,10,20$, and $50 \mathrm{G}$. Figure 1(a) shows schematically one of these masks which results in the formation of CDs only within the circular apertures of about $90 \mu \mathrm{m}$ diam. This patterning allows very sensitive comparison of the vortex structure and the melting processes in adjacent irradiated and pristine regions, which is not possible by other methods.

In the absence of CDs the vortices form quasiordered lattice with no topological defects (Bragg glass) [4], as seen in the pristine part of the magnetic decoration image in Figs. 1(b) and 1(c). What happens to this phase when sparse CDs are added? The irradiated region in Fig. 1(b)

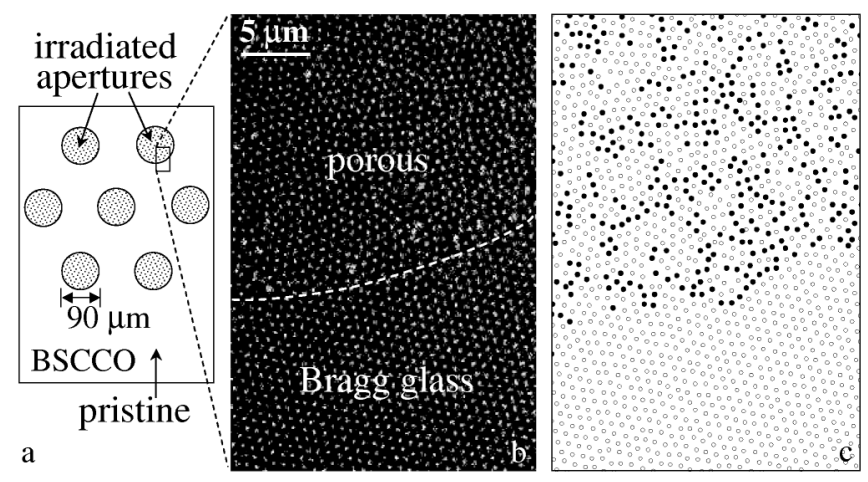

FIG. 1. (a) Schematic of BSCCO crystal irradiated through a mask with an array of circular apertures. (b) Bitter decoration image ( $B=40 \mathrm{G}, T=4.2 \mathrm{~K}$ ) showing pristine region at the bottom (Bragg glass) and a section of the irradiated region $\left(B_{\phi}=10 \mathrm{G}\right)$ on top where porous vortex matter is formed. (c) Corresponding locations of sixfold (open circles) and of five- and sevenfold coordinated vortices (solid circles) obtained by Delaunay triangulation. 
shows that it is no longer Bragg glass since it has significant amount of topological defects [solid circles in Fig. 1(c)] and no orientational long-range order [5]. It is also not an amorphous or glass phase in the usual sense, nor is it a simple polycrystal as discussed below. In the presence of CDs the Bose glass (BG) theory [6-8] is usually applied, which describes the vortex matter in terms of anisotropic homogeneously pinned medium. Such a description is adequate for the common situation of high irradiation doses $\left(B_{\phi}>B\right)$, in which all the vortices reside on CDs and the vortex pinning energies are comparable. In contrast, we investigate here mainly the opposite extreme of low doses in which the vortices greatly outnumber the CDs, i.e., $B \gg B_{\phi}$. In this case the system is inherently heterogeneous, consisting of two vortex populations with well separated characteristic energies: The vortices residing on CDs are strongly pinned and form a rigid network or matrix, whereas the interstitial vortices are localized by significantly weaker elastic interactions and form relatively soft crystallites within the pores of the matrix. In the following we refer to this state [upper parts of Figs. 1(b) and 1(c)] as porous vortex matter in order to emphasize the important consequences of the heterogeneous structure.

Figure 2 shows several frames from a "movie" [9] of the melting process at indicated temperatures $T$ at two fields, 30 and $60 \mathrm{G}$. Each frame is obtained by taking the difference between the MO images at $T+0.15 \mathrm{~K}$ and $T-0.15 \mathrm{~K}$ and averaging a large number of such differential images [3]. The bright features show the regions in the sample that undergo a FOT within the temperature interval of $0.3 \mathrm{~K}$, and the intensity of this bright paramagnetic signal is the equilibrium magnetization step $\Delta B$ at the transition $[3,10]$. Figure 2(a) shows the nucleation of the liquid phase in the form of bright inclined strips in the central pristine region which arise from intrinsic

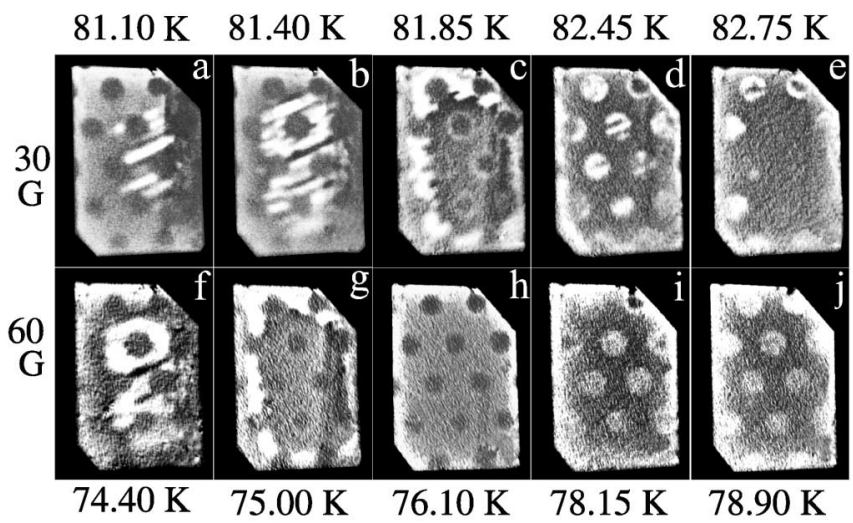

FIG. 2. Differential MO images of the melting process vs $T$ at fields of 30 and $60 \mathrm{G}$ in BSCCO crystal $0.65 \times 0.45 \times$ $0.01 \mathrm{~mm}^{3}$. The bright regions are the areas that undergo melting within $T$ modulation of $0.3 \mathrm{~K}$. (a)-(c) and (f) $-(\mathrm{h})$ : melting of the pristine regions while the irradiated apertures are still solid. (d), (e), (i), and ( $\mathrm{j}$ ): melting of the irradiated apertures $\left(B_{\phi}=20 \mathrm{G}\right)$. The area outside the crystal is blackened [20]. sample disorder (see [3]). With increasing $T$ [Fig. 2(b)] the liquid expands, remarkably avoiding the irradiated apertures. In Fig. 2(c) the entire central pristine part of the sample is liquid, while the apertures with $B_{\phi}=20 \mathrm{G}$ are still solid. In Fig. 2(d) the central apertures melt at $82.45 \mathrm{~K}$, which is about $1 \mathrm{~K}$ above $T_{m}$ of the adjacent pristine regions in Fig. 2(b). The apertures closer to the sample edges begin to melt in Fig. 2(e). The FOT is equally strong in the irradiated and pristine regions: The $\Delta B$ step derived from the paramagnetic melting signal [10] is the same in Figs. 2(b) and 2(d). Also the width of the local melting transition is the same within our resolution, i.e., each point in the sample melts within $0.3 \mathrm{~K}$ or less. This is the first direct observation of an upward shift of the FOT by correlated disorder.

The melting process at $60 \mathrm{G}$ (Fig. 2, second row) reveals two important differences. First, the shift of the melting temperature, $\Delta T_{m}$, is about $4 \mathrm{~K}$ [difference between Figs. 2(f) and 2(i)] which is much larger as compared to $1 \mathrm{~K}$ at $30 \mathrm{G}$. In Fig. 2(h), for example, the entire pristine sample has melted while the irradiated apertures are still solid. Second, the brightness of the paramagnetic melting signal $\Delta B$ in the apertures in Fig. 2(i) is much lower than in the pristine sample, and moreover, the melting in each aperture is broadened over several frames, as exemplified in Fig. 2(j). At still higher fields, above $100 \mathrm{G}$, no paramagnetic FOT signal is detected in the irradiated apertures with $B_{\phi}=20 \mathrm{G}$.

We have also used differential MO with field modulation of $1 \mathrm{G}$ (Fig. 3) instead of the $T$ modulation. In addition to detecting the FOT [3], this method provides a very sensitive measurement of the irreversibility line at a very low effective frequency of about $0.1 \mathrm{~Hz}$. Figure 3 demonstrates the determination of the irreversibility line in the region where no FOT is present. In Fig. 3(a) the entire pristine sample is in the liquid state, while the apertures are still solid. The apertures appear black, showing that the external field modulation is shielded due to the enhanced pinning. Upon increasing the field

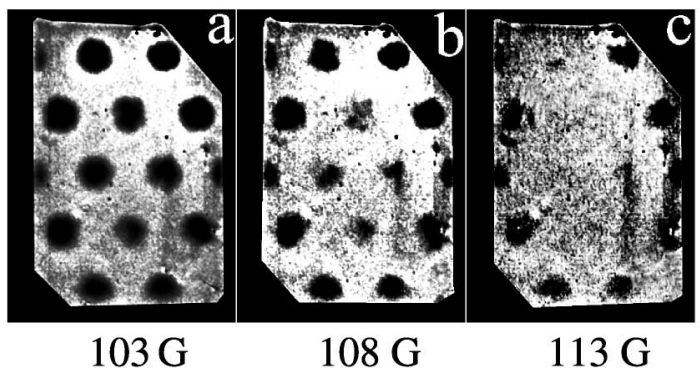

FIG. 3. Differential MO images using field modulation of $1 \mathrm{G}$ in BSCCO crystal of Fig. 2 at three fields at $T=68.4 \mathrm{~K}$. (a) All the pristine regions are liquid while the irradiated apertures $\left(B_{\phi}=20 \mathrm{G}\right)$ are still solid and irreversible, and hence appear black due to shielding. (b) Partial reversibility of the central apertures. (c) Central apertures are liquid while the apertures closer to the edges begin to melt. 
the black apertures disappear [Figs. 3(b) and 3(c)] revealing the value of the local irreversibility field.

Figure 4 shows the location of the onset of the FOT for $B_{\phi}=5,10,20$, and $50 \mathrm{G}$ obtained by $T$ modulation (solid symbols) and of the irreversibility line obtained by field modulation (open symbols). The solid lines are guides to the eye for the FOT lines which terminate at the corresponding critical points (CPs). The irreversibility data coincide with the FOT line below the CP and smoothly extrapolate the location of the transition line to higher fields. The first interesting observation here is that although the structures of the porous vortex matter and of the Bragg glass are very different (Fig. 1), their phase diagrams for $B_{\phi}=5 \mathrm{G}$ in Fig. 4 are almost identical, with a slight upward shift of the FOT. This brings us to an important conclusion that the quasi-long-range order that characterizes the Bragg glass is apparently not an essential requirement for the existence of a FOT [11].

We can understand the upward shift in $B_{m}(T)$ in Fig. 4 by generalizing the cage model concept. In a pure system each vortex is confined in an elastic potential cage of its neighbors, and melting occurs when the transverse thermal fluctuations $\left\langle u_{T}\right\rangle$ reach a certain fraction $c_{L}$ of the lattice spacing $a_{0}$. The pores of the matrix are vertical cylinders which provide an additional confining cage potential to the interstitial vortices. This enhanced rigidity reduces $\left\langle u_{T}\right\rangle$, and hence stabilizes the solid crystallites within the pores, thereby shifting $B_{m}(T)$ upwards. The shift in $B_{m}(T)$ grows with $B_{\phi}$ due to the decrease in the size of the pores. Since the pinning energy of the CDs is usually significantly larger than the elastic energy, the melting of the crystallites may occur without the destruction of the matrix, hence forming an interstitial liquid

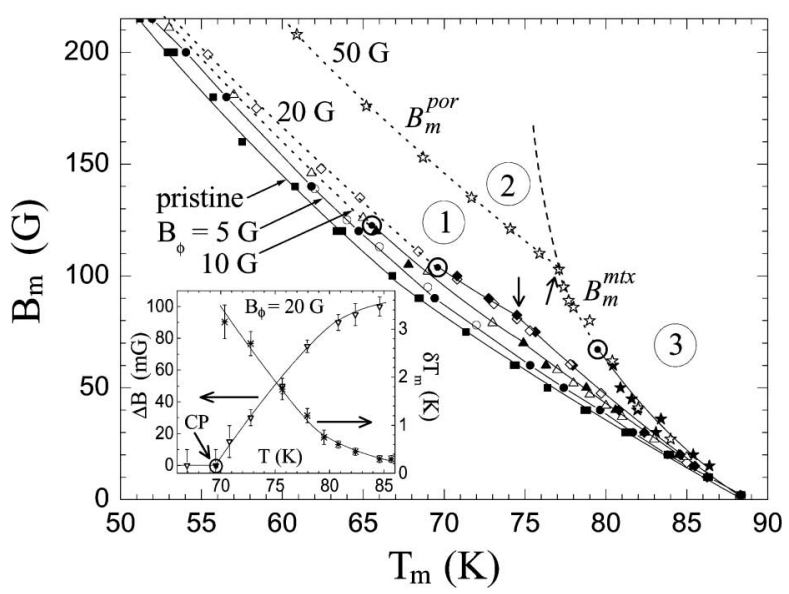

FIG. 4. The melting lines $B_{m}(T)$ of the pristine and irradiated regions with indicated $B_{\phi}$. Solid (open) symbols are temperature (field) modulation data showing the location of the FOT (irreversibility line). Solid (dotted) lines are guides to the eye of the first-order (continuous) transitions that terminate at the critical points $\odot$. Inset: The height of the FOT equilibrium magnetization step $\Delta B$ which vanishes at the $C P$, and the local width $\delta T_{m}$ of the FOT vs $T$. within a rigid matrix as predicted theoretically [8] and in numerical simulations [12].

Upon increasing the density of CDs the FOT is weakened and it eventually transforms into a continuous transition in Fig. 4. This transformation is seen more clearly by varying the temperature along a given $B_{m}(T)$ line. The inset to Fig. 4 shows the height of the equilibrium magnetization step $\Delta B$ vs $T$ for $B_{\phi}=20 \mathrm{G}$. As $T$ is decreased $\Delta B$ drops and vanishes at a CP below which no discontinuity is found and the melting becomes continuous. In addition, the width of the FOT, $\delta T_{m}$, shows a significant broadening on approaching the $\mathrm{CP}$ (Fig. 4 inset, right axis). This indicates that it is a true $\mathrm{CP}$ at which the FOT transforms into a continuous transition as expected theoretically [13]. This $\mathrm{CP}$ is unlike the apparent pointdisorder-induced $\mathrm{CP}$ in $\mathrm{BSCCO}$ at lower $T$ where no appreciable broadening was observed [14], and where the FOT was found to be obscured by pinning-induced irreversibility in the liquid phase [15]. Here, in contrast, the liquid is fully reversible and no such experimental limitation exists. Figure 4 shows that the $\mathrm{CP}$ is shifted to lower fields with $B_{\phi}$ [16]. Note that in YBCO the CP was observed to shift upward with CDs [17]. There, however, the $\mathrm{CP}$ is induced by the pristine point disorder and the CDs just slightly modify its position by suppressing vortex meandering. In our case, in contrast, the CDs apparently create a new $\mathrm{CP}$ that does not exist in pristine crystals.

We now discuss another key finding which is a kink in the $B_{m}(T)$ lines marked by the arrows in Fig. 4. This kink becomes very prominent when the temperature shift $\Delta T_{m}$ between the irradiated and pristine melting lines is plotted as shown in Fig. 5. The inset displays the corresponding upward shift in field $\Delta B_{m}$. In BG theory a kink in the transition line is expected to occur at $B_{k} \approx B_{\phi}[7,8]$. We argue that the kink in Fig. 5 is a new feature of a completely different nature, which reflects the collapse of the rigid matrix. The BG kink is experimentally found

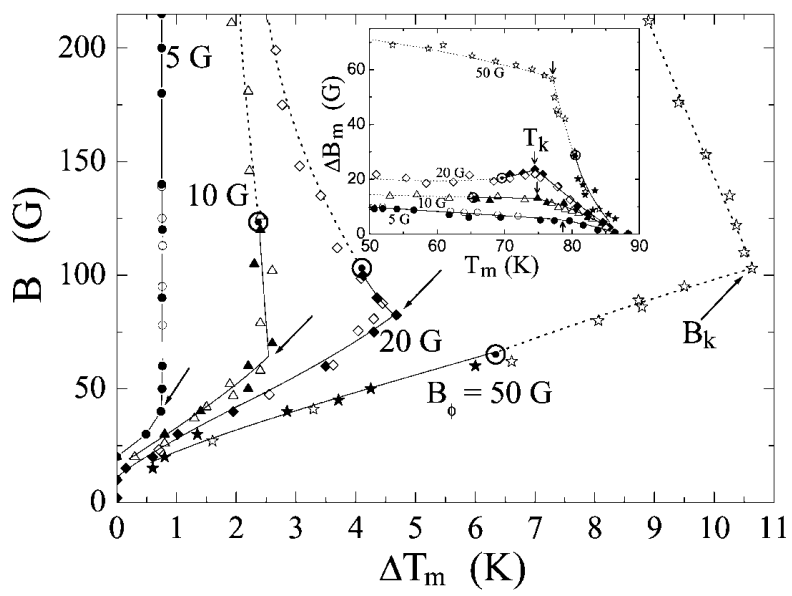

FIG. 5. The shift $\Delta T_{m}$ in the melting temperature for different $B_{\phi}$ with respect to the pristine $T_{m}$ at various fields $B$. Inset: The upward shift $\Delta B_{m}$ in the melting field vs $T_{m}$. 
to occur at $B_{k} / B_{\phi}$ of $1 / 6$ to 1 for large $B_{\phi}$, and $B_{k}$ usually scales linearly with $B_{\phi}[18,19]$. The kink in Fig. 5 occurs in the opposite regime of $B_{k} / B_{\phi} \approx 2$ to 8 , and it does not scale with $B_{\phi}$. The BG kink is a broad feature that was found to occur only along a glass transition line [18,19]. In contrast, here the kink is extremely sharp, and it is the first observation of a kink that occurs along a FOT line. Finally, the BG kink is a transition from a strong influence of the CDs below $B_{k}$ to a much weaker effect of CDs above it $[7,8]$. Here, in contrast, the situation is just the opposite, as seen in the inset to Fig. 5. The effect of CDs is large at fields above $B_{k}$, where the shift $\Delta B_{m}$ is almost constant and approximately equal to $B_{\phi}$, and it collapses rapidly at $T>T_{k}$. This collapse occurs at $T_{k} \simeq 75 \mathrm{~K}$, with little dependence on $B_{\phi}$. Interestingly, the kink occurs along the FOT line for $B_{\phi} \leq 20 \mathrm{G}$, while for $B_{\phi}=50 \mathrm{G}$ it falls in the region of continuous transition.

In Fig. 4 three regions are enumerated with respect to $B_{\phi}=50 \mathrm{G}$ curve. In region 1 the crystallites in the pores are stabilized by the rigid matrix and melt at $B_{m}^{\text {por }}$, well above the pristine $B_{m}(T)$. In region 2 the pores are liquid while the matrix remains intact, and hence $B_{m}^{\text {por }}$ reflects a melting transition of the softer of the two substances in a heterogeneous medium. This unconventional melting cannot be described by a single set of parameters since it depends on the properties of both the pores and the matrix, each having different field and temperature dependencies. As a result, $B_{m}^{\text {por }}(T)$ does not extrapolate to $T_{c}$, but rather well above it, which is a very uncommon behavior at high temperatures. This heterogeneous process collapses sharply at $T_{k}$, above which the matrix delocalizes, resulting in a homogeneous liquid in region 3. The $B_{m}^{\mathrm{mtx}}$ line thus describes the melting process of the matrix due to depinning from the CDs, which leads to immediate melting of the superheated crystallites. Thus in contrast to the common homogeneous BG description, the $B_{m}^{\mathrm{por}}(T)$ and $B_{m}^{\mathrm{mtx}}(T)$ lines originate from two independent heterogeneous processes, melting of crystallites within a rigid matrix and the collapse of the matrix itself, resulting in a sharp kink at the intersection point. We expect the $B_{m}^{\mathrm{mtx}}(T)$ line to extend into the liquid phase [8], like the dashed line in Fig. 4, separating the interstitial liquid region 2 from the homogeneous liquid 3. Our MO measurements cannot detect this line since both these regions are fully reversible. In region 2 , however, we expect a higher $c$ axis correlation than in region 3, which is apparently consistent with the recently reported recoupling transition [19].

In summary, when vortices outnumber CDs, heterogeneity rather than the average properties of the lattice has to be taken into account for a proper description of the structure and the thermodynamic behavior of the vortex matter. We have found evidence for two mechanisms: melting of superheated crystallites within the pores of a solid matrix and the destruction of the rigid matrix. The intersection point of these two independent processes results in a sharp kink in the observed melting line. The heterogeneous melting can be either first order or continuous, depending on temperature and the density of CDs. The porous vortex matter may thus provide a tunable model system for general comprehension of melting of nanocrystals in porous materials.

This work was supported by the Israel Science Foundation and Center of Excellence Program, by the German-Israeli Foundation GIF, by the Minerva Foundation, Germany, and by the Grant-in-Aid for Scientific Research from the Ministry of Education, Science, Sports and Culture, Japan. E. Z. and F. C. acknowledge support by the Fundacion Antorchas-WIS Collaboration Program.

*Present address: Bell Labs, Lucent Technologies, Murray Hill, New Jersey 07974.

[1] P. R. Couchman and W. A. Jesser, Nature (London) 269, 481 (1977); A. N. Goldstein, C. M. Echer, and A. P. Alivisatos, Science 256, 1425 (1992).

[2] L. Gråbaek et al., Phys. Rev. Lett. 64, 934 (1990); H.W. Sheng et al., Philos. Mag. Lett. 73, 179 (1996); Q. Jiang, Z. Zhang, and J. C. Li, Chem. Phys. Lett. 322, 549 (2000).

[3] A. Soibel et al., Nature (London) 406, 282 (2000); A. Soibel et al., Phys. Rev. Lett. 87, 167001 (2001); M. Yasugaki et al., Phys. Rev. B 65, 212502 (2002).

[4] T. Giamarchi and P. Le Doussal, Phys. Rev. Lett. 72, 1530 (1994).

[5] M. Menghini et al., cond-mat/0210623.

[6] D. R. Nelson and V. M. Vinokur, Phys. Rev. Lett. 68, 2398 (1992).

[7] A. I. Larkin and V. M. Vinokur, Phys. Rev. Lett. 75, 4666 (1995).

[8] L. Radzihovsky, Phys. Rev. Lett. 74, 4923 (1995).

[9] See EPAPS Document No. E-PRLTAO-90-058307 for color movies of melting at 30 and $50 \mathrm{G}$. A direct link to this document may be found in the online article's HTML reference section. The document may also be reached via the EPAPS homepage (http://www.aip.org/ pubservs/epaps.html) or from ftp.aip.org in the directory lepaps/. See the EPAPS homepage for more information.

[10] N. Morozov et al., Phys. Rev. B 54, R3784 (1996).

[11] S. Colson et al., cond-mat/0204493.

[12] P. Sen, N. Trivedi, and D. M. Ceperley, Phys. Rev. Lett. 86, 4092 (2001); Y. Nonomura and X. Hu, cond-mat/ 0212609 .

[13] Y. Imry and M. Wortis, Phys. Rev. B 19, 3580 (1979).

[14] E. Zeldov et al., Nature (London) 375, 373 (1995).

[15] N. Avraham et al., Nature (London) 411, 451 (2001).

[16] B. Khaykovich et al., Phys. Rev. B 57, R14088 (1998).

[17] W. K. Kwok et al., Phys. Rev. Lett. 84, 3706 (2000).

[18] C. J. van der Beek et al., Phys. Rev. Lett. 86, 5136 (2001); L. Krusin-Elbaum et al., Phys. Rev. Lett. 72, 1914 (1994).

[19] M. Sato et al., Phys. Rev. Lett. 79, 3759 (1997); T. Tamegai et al., in Advances in Superconductivity XI: Proceedings of the 11th International Symposium on Superconductivity (ISS'98), 1998, Fukuoka (SpringerVerlag, Tokyo, 1999), p. 571.

[20] Color MO movies are available at http://www.weizmann. ac.il/home/fnsup/ 